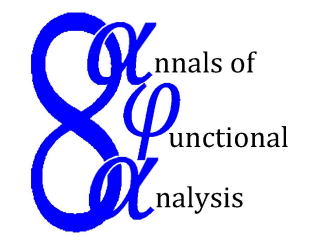

Ann. Funct. Anal. 6 (2015), no. 2, 104-115

http://doi.org/10.15352/afa/06-2-10

ISSN: 2008-8752 (electronic)

http://projecteuclid.org/afa

\title{
INNER FUNCTIONS AND WEIGHTED COMPOSITION OPERATORS ON THE HARDY-HILBERT SPACE WITH THE UNBOUNDED WEIGHTS
}

\author{
KEI JI IZUCHI ${ }^{*}$, KOU HEI IZUCHI ${ }^{2}$ AND YUKO IZUCHI ${ }^{3}$ \\ Communicated by J. Esterle
}

\begin{abstract}
Let $\varphi$ be an analytic self-map of the open unit disk. It is given several sufficient conditions on $\varphi$ for which there is $u \in H^{2} \backslash H^{\infty}$ such that the weighted composition operator $M_{u} C_{\varphi}$ on $H^{2}$ is bounded.
\end{abstract}

\section{INTRODUCTION}

Let $\mathbb{D}$ be the open unit disk and $m$ be the normalized Lebesgue measure on $\partial \mathbb{D}$. We denote by $L^{2}(\partial \mathbb{D})$ the space of square integrable functions on $\partial \mathbb{D}$ with respect to $m$. For $1 \leq p<\infty$, let $H^{p}$ be the space of analytic functions $f$ on $\mathbb{D}$ satisfying

$$
\|f\|_{p}^{p}:=\lim _{r \rightarrow 1} \int_{\partial \mathbb{D}}\left|f\left(r e^{i \theta}\right)\right|^{p} d m\left(e^{i \theta}\right)<\infty .
$$

The space $H^{p}$ is called the Hardy space. We denote by $H^{\infty}$ the space of bounded analytic functions on $\mathbb{D}$ with the supremum norm $\|f\|_{\infty}$. For each $f \in H^{2}$, there is the boundary function $f^{*}$ of $f$ defined by $f^{*}\left(e^{i \theta}\right)=\lim _{r \rightarrow 1} f\left(r e^{i \theta}\right)$ a.e. on $\partial \mathbb{D}$. We have $f^{*} \in L^{2}(\partial \mathbb{D})$ (see $\left.[2,3,4]\right)$.

We denote by $\mathcal{S}$ the set of analytic self-maps of $\mathbb{D}$. For each $\varphi \in \mathcal{S}$, we may define the composition operator $C_{\varphi}$ by $C_{\varphi} f=f \circ \varphi$ for $f \in H^{2}$. By the Littlewood subordination theorem [6], $C_{\varphi}$ is a bounded linear operator on $H^{2}$. Recently there are many researches on composition operators on various spaces of analytic functions. For $u \in H^{\infty}$, we may define the weighted composition

Date: Received: Jun. 1, 2014; Accepted: Jul. 13, 2014.

* Corresponding author.

2010 Mathematics Subject Classification. Primary 47B38; Secondary 30H10.

Key words and phrases. Hardy space, inner function, weighted composition operator, boundedness. 
operator $M_{u} C_{\varphi}: H^{2} \rightarrow H^{2}$ by $\left(M_{u} C_{\varphi}\right) f=u(f \circ \varphi)$. Of course, $M_{u} C_{\varphi}$ is bounded on $H^{2}$. See $[1,9]$ for the basic properties of (weighted) composition operators.

Let $u \in H^{2}$ and $\varphi \in \mathcal{S}$. For each $f \in H^{2}$, we have

$$
\left\|M_{u} C_{\varphi} f\right\|_{1} \leq\|u\|_{2}\left\|C_{\varphi} f\right\|_{2} \leq\|u\|_{2}\left\|C_{\varphi}\right\|\|f\|_{2}
$$

Hence $M_{u} C_{\varphi}: H^{2} \rightarrow H^{1}$ is a bounded linear map. If $\|\varphi\|_{\infty}<1$, then it is not difficult to see that $M_{u} C_{\varphi}: H^{2} \rightarrow H^{2}$ is bounded.

In this paper, for $\varphi \in \mathcal{S}$ with $\|\varphi\|_{\infty}=1$ we shall study the boundedness of $M_{u} C_{\varphi}: H^{2} \rightarrow H^{2}$ (see $[5,8]$ ). More precisely, we consider the following problem.

Problem 1.1. For which $\varphi \in \mathcal{S}$, is there $u \in H^{2} \backslash H^{\infty}$ such that $M_{u} C_{\varphi}: H^{2} \rightarrow H^{2}$ is bounded?

A function $\psi \in H^{\infty}$ is called inner if $\left|\psi^{*}\right|=1$ a.e. on $\partial \mathbb{D}$. In [5, Corollary 2.2], Nguyen, Ohno and the first author showed that if $\varphi \in \mathcal{S}$ is not inner, then there is $u \in H^{2} \backslash H^{\infty}$ such that $M_{u} C_{\varphi}: H^{2} \rightarrow H^{2}$ is bounded. So mainly we assume that $\varphi$ is an inner function. We denote by $\operatorname{supp}(\varphi)$ the set of $e^{i \theta} \in \partial \mathbb{D}$ at which $\varphi$ does not have a continuous extension. Then $\operatorname{supp}(\varphi)$ is a closed subset of $\partial \mathbb{D}$. It is known that $\operatorname{supp}(\varphi)=\emptyset$ if and only if $\varphi$ is a finite Blaschke product. It is not difficult to see that if $\operatorname{supp}(\varphi)=\emptyset$, then $M_{u} C_{\varphi}: H^{2} \rightarrow H^{2}$ is unbounded for every $u \in H^{2} \backslash H^{\infty}$ (see [5, p. 1335]).

It is known that $\varphi$ may be extended to a non-vanishing analytic function on some neighborhood of each $e^{i \theta} \in \partial \mathbb{D} \backslash \operatorname{supp}(\varphi)$. Hence we may think that $\varphi^{*}$ is differentiable on $\partial \mathbb{D} \backslash \operatorname{supp}(\varphi)$. In [5, Proposition 2.9], Nguyen, Ohno and the first author essentially proved that if $\sup _{z \in \partial \mathbb{D} \backslash \operatorname{supp}(\varphi)}\left|\varphi^{* \prime}(z)\right|=\infty$, then there is $u \in H^{2} \backslash H^{\infty}$ such that $M_{u} C_{\varphi}: H^{2} \rightarrow H^{2}$ is bounded.

In Section 2, we shall prove that if there is an open subarc $U$ of $\partial \mathbb{D}$ such that $U \cap \operatorname{supp}(\varphi) \neq \emptyset$ and $U \cap \operatorname{supp}(\varphi)$ does not contain any interior points, then $\sup _{z \in \partial \mathbb{D} \backslash \operatorname{supp}(\varphi)}\left|\varphi^{* \prime}(z)\right|=\infty$, so there exists a function $u \in H^{2} \backslash H^{\infty}$ such that $M_{u} C_{\varphi}: H^{2} \rightarrow H^{2}$ is bounded.

For an inner function $\varphi$, we consider the following two conditions.

$(\alpha)$ There is a sequence of mutually disjoint measurable subsets $\left\{C_{n}\right\}_{n \geq 1}$ of $\partial \mathbb{D}$ and a sequence of positive numbers $\left\{\delta_{n}\right\}_{n \geq 1}$ satisfying $\sum_{n=1}^{\infty} \delta_{n}<\infty$ such that $m\left(C_{n}\right)>0$ and $m\left(C_{n} \cap \varphi^{*(-1)}(E)\right) \leq \delta_{n} m(E)$ for every measurable subset $E$ of $\partial \mathbb{D}$ and for every $n \geq 1$.

$(\beta)$ There is a sequence of mutually disjoint measurable subsets $\left\{E_{n}\right\}_{n \geq 1}$ of $\partial \mathbb{D}$ such that $m\left(E_{n} \cap \varphi^{*(-1)}(E)\right)>0$ for every measurable subset $E$ of $\partial \mathbb{D}$ satisfying $m(E)>0$ and for every $n \geq 1$.

We do not know whether conditions $(\alpha)$ and $(\beta)$ hold or not for every inner function $\varphi$ satisfying $m(\operatorname{supp}(\varphi))>0$. In Section 3, we shall prove that if an inner function $\varphi$ satisfies condition $(\alpha)$, then there is $u \in H^{2} \backslash H^{\infty}$ such that $M_{u} C_{\varphi}: H^{2} \rightarrow H^{2}$ is bounded. We also show that if $\varphi$ satisfies condition $(\beta)$, then $\varphi$ satisfies condition $(\alpha)$.

The techniques used here will give us some light on further study of Problem 1.1. 


\section{Bounded WEIGHTED COMPOSITION OPERATORS}

The following proposition was proven in [5, Corollary 2.2]. We shall give its another proof.

Proposition 2.1. Let $\varphi \in \mathcal{S}$. If $\varphi$ is not inner, then there is $u \in H^{2} \backslash H^{\infty}$ such that $M_{u} C_{\varphi}: H^{2} \rightarrow H^{2}$ is bounded.

Proof. For $0<r<1$, we write $\left\{\left|\varphi^{*}\right|<r\right\}=\left\{e^{i t} \in \partial \mathbb{D}:\left|\varphi^{*}\left(e^{i t}\right)\right|<r\right\}$. Take $0<r<1$ satisfying $m\left(\left\{\left|\varphi^{*}\right|<r\right\}\right)>0$. Let $\eta$ be a positive unbounded function in $L^{2}(\partial \mathbb{D})$ such that $\eta=1$ on $\partial \mathbb{D} \backslash\left\{\left|\varphi^{*}\right|<r\right\}$ and $\eta \geq 1$ a.e. on $\left\{\left|\varphi^{*}\right|<r\right\}$. By [4, p. 53], there is $u \in H^{2}$ satisfying $\left|u^{*}\right|=\eta$ a.e. on $\partial \mathbb{D}$. We have $u \notin H^{\infty}$. For $f \in H^{2}$, by [2, p. 36] we have

$$
|f(z)| \leq \frac{\sqrt{2}\|f\|_{2}}{\sqrt{1-r}}, \quad|z| \leq r .
$$

Hence

Therefore

$$
\left|f\left(\varphi^{*}\left(e^{i \theta}\right)\right)\right| \leq \frac{\sqrt{2}\|f\|_{2}}{\sqrt{1-r}}, \quad e^{i \theta} \in\left\{\left|\varphi^{*}\right|<r\right\} .
$$

$$
\begin{aligned}
& \left\|M_{u} C_{\varphi} f\right\|_{2}^{2} \\
& =\int_{\partial \mathbb{D}}\left|u^{*}\right|^{2}\left|(f \circ \varphi)^{*}\right|^{2} d m \\
& =\int_{\left\{\left|\varphi^{*}\right|<r\right\}}\left|u^{*}\right|^{2}\left|(f \circ \varphi)^{*}\right|^{2} d m+\int_{\partial \mathbb{D} \backslash\left\{\left|\varphi^{*}\right|<r\right\}}\left|u^{*}\right|^{2}\left|(f \circ \varphi)^{*}\right|^{2} d m \\
& \leq \frac{2\|f\|_{2}^{2}}{1-r} \int_{\partial \mathbb{D}}\left|u^{*}\right|^{2} d m+\int_{\partial \mathbb{D}}\left|(f \circ \varphi)^{*}\right|^{2} d m \\
& \leq\left(\frac{2\|\eta\|_{2}^{2}}{1-r}+\left\|C_{\varphi}\right\|^{2}\right)\|f\|_{2}^{2} .
\end{aligned}
$$

Thus $M_{u} C_{\varphi}: H^{2} \rightarrow H^{2}$ is bounded.

Hereafter, to study Problem 1.1 we assume that $\varphi$ is an inner function satisfying $\operatorname{supp}(\varphi) \neq \emptyset$. In [5, Proposition 2.9], Nguyen, Ohno and the first author proved the following essentially.

Lemma 2.2. Let $\varphi$ be an inner function. If $\sup _{z \in \partial \mathbb{D} \backslash \operatorname{supp}(\varphi)}\left|\varphi^{* \prime}(z)\right|=\infty$, then there exists a function $u \in H^{2} \backslash H^{\infty}$ such that $M_{u} C_{\varphi}: H^{2} \rightarrow H^{2}$ is bounded.

Let $\varphi$ be an inner function and $I=\left\{e^{i t}: t_{1}<t<t_{2}\right\}$ satisfy $I \cap \operatorname{supp}(\varphi)=\emptyset$. Then there is a real valued differentiable function $\sigma(t)$ such that $\varphi^{*}\left(e^{i t}\right)=e^{i \sigma(t)}$ and $\sigma^{\prime}(t)>0$ on $\left(t_{1}, t_{2}\right)$. Admitting the values $\infty,-\infty$, we may define $s_{k}=$ $\lim _{t \rightarrow t_{k}} \sigma(t)$ for $k=1,2$. Putting $\sigma\left(t_{k}\right)=s_{k}$, we think $\sigma(t)$ of an extended real valued continuous function on $\left[t_{1}, t_{2}\right]$ and $\varphi^{*}(I)=\left\{e^{i s}: s_{1}<s<s_{1}\right\}$.

For $e^{i t_{0}} \in \partial \mathbb{D}$ and $\varepsilon>0$, we write $I_{\varepsilon}\left(e^{i t_{0}}\right)=\left\{e^{i t}: t_{0}-\varepsilon<t<t_{0}+\varepsilon\right\}$.

Lemma 2.3. Let $\varphi$ be an inner function. If $\operatorname{supp}(\varphi)=\left\{e^{i t_{0}}\right\}$, then

$$
\sup _{z \in I_{\varepsilon}\left(e^{i t_{0}}\right) \backslash\left\{e^{i t_{0}}\right\}}\left|\varphi^{* \prime}(z)\right|=\infty
$$


for every $\varepsilon>0$.

Proof. There is a real valued differentiable function $\sigma(t)$ on $\left(t_{0}, t_{0}+2 \pi\right)$ such that $\varphi^{*}\left(e^{i t}\right)=e^{i \sigma(t)}$ and $\sigma^{\prime}(t)>0$ for every $t_{0}<t<t_{0}+2 \pi$. Then either $\lim _{t \rightarrow t_{0}} \sigma(t)=-\infty$ or $\lim _{t \rightarrow t_{0}+2 \pi} \sigma(t)=\infty$ (see [3, p. 90-91]). Hence we get the assertion.

Lemma 2.4. Let $\varphi_{1}, \varphi_{2}$ be inner functions and $I$ be an open subarc of $\partial \mathbb{D}$ such that $I \cap \operatorname{supp}\left(\varphi_{1} \varphi_{2}\right)=\emptyset$. Then $\left|\left(\varphi_{1} \varphi_{2}\right)^{* \prime}\right|=\left|\varphi_{1}^{* \prime}\right|+\left|\varphi_{2}^{* \prime}\right|$ on $I$.

Proof. Let $I=\left\{e^{i t}: t_{1}<t<t_{2}\right\}$. There are real valued differentiable functions $\sigma_{1}(t), \sigma_{2}(t)$ on $\left(t_{1}, t_{2}\right)$ such that $\varphi_{1}^{*}\left(e^{i t}\right)=e^{i \sigma_{1}(t)}, \varphi_{2}^{*}\left(e^{i t}\right)=e^{i \sigma_{2}(t)}, \sigma_{1}^{\prime}(t)>0$ and $\sigma_{2}^{\prime}(t)>0$ for every $t_{1}<t<t_{2}$. We have $\left(\varphi_{1} \varphi_{2}\right)^{*}\left(e^{i t}\right)=e^{i\left(\sigma_{1}(t)+\sigma_{2}(t)\right)}$. Hence

$$
\left(\varphi_{1} \varphi_{2}\right)^{* \prime}\left(e^{i t}\right)=-i e^{-i t} \frac{d}{d t}\left(\varphi_{1} \varphi_{2}\right)^{*}\left(e^{i t}\right)=e^{-i t}\left(\sigma_{1}^{\prime}(t)+\sigma_{2}^{\prime}(t)\right) e^{i\left(\sigma_{1}(t)+\sigma_{2}(t)\right)}
$$

for $t_{1}<t<t_{2}$. Therefore

$$
\left|\left(\varphi_{1} \varphi_{2}\right)^{* \prime}\left(e^{i t}\right)\right|=\sigma_{1}^{\prime}(t)+\sigma_{2}^{\prime}(t)=\left|\varphi_{1}^{* \prime}\left(e^{i t}\right)\right|+\left|\varphi_{2}^{* \prime}\left(e^{i t}\right)\right| .
$$

For a subset $E$ of $\partial \mathbb{D}$, we denote by int $E$ the interior of $E$ in $\partial \mathbb{D}$.

Theorem 2.5. Let $\varphi$ be an inner function. If $\operatorname{supp}(\varphi) \neq \overline{\text { int } \operatorname{supp}(\varphi)}$, then $\sup _{z \in \partial \mathbb{D} \backslash \operatorname{supp}(\varphi)}\left|\varphi^{* \prime}(z)\right|=\infty$.

Proof. Take $e^{i t_{0}} \in \operatorname{supp}(\varphi) \backslash \overline{\operatorname{int} \operatorname{supp}(\varphi)}$ and then take an open subarc $I$ of $\partial \mathbb{D}$ such that $e^{i t_{0}} \in I$ and $I \cap \overline{\operatorname{int} \operatorname{supp}(\varphi)}=\emptyset$. For each $\lambda \in \mathbb{D}$, let $\tau_{\lambda}(z)=$ $(z-\lambda) /(1-\bar{\lambda} z), z \in \mathbb{D}$. By Frostman's theorem (see [3, p. 79]), there is $\lambda \in \mathbb{D}$ such that $\psi:=\tau_{\lambda} \circ \varphi$ is a Blaschke product. We have $\operatorname{supp}(\psi)=\operatorname{supp}(\varphi)$. Then there is a sequence $\left\{a_{k}\right\}_{k \geq 1}$ in $\mathbb{D}$ such that $\psi\left(a_{k}\right)=0$ for every $k \geq 1$ and $a_{k} \rightarrow e^{i t_{0}}$ as $k \rightarrow \infty$. Let $\psi_{1}$ be the Blaschke subproduct of $\psi$ with zeros $\left\{a_{k}\right\}_{k \geq 1}$. Then $\operatorname{supp}\left(\psi_{1}\right)=\left\{e^{i t_{0}}\right\}$. Let $\psi_{2}=\psi / \psi_{1}$. Retaking a further subsequence of $\left\{a_{k}\right\}_{k \geq 1}$, we may assume that $\operatorname{supp}\left(\psi_{2}\right)=\operatorname{supp}(\psi)$. Since $e^{i t_{0}} \in I$, we may take $\varepsilon>0$ satisfying

$$
I_{\varepsilon}\left(e^{i t_{0}}\right)=\left\{e^{i t}: t_{0}-\varepsilon<t<t_{0}+\varepsilon\right\} \subset I .
$$

By Lemma 2.3, we have $\sup _{z \in I_{\varepsilon}\left(e^{i t_{0}}\right) \backslash\left\{e^{i t_{0}}\right\}}\left|\psi_{1}^{* \prime}(z)\right|=\infty$. Since $I \cap \overline{\text { int } \operatorname{supp}(\psi)}=\emptyset$, $I_{\varepsilon}\left(e^{i t_{0}}\right) \backslash \operatorname{supp}(\psi)$ is dense in $I_{\varepsilon}\left(e^{i t_{0}}\right)$. Hence

$$
\sup _{z \in I_{\varepsilon}\left(e^{i t_{0}}\right) \backslash \operatorname{supp}(\psi)}\left|\psi_{1}^{* \prime}(z)\right|=\infty .
$$

Therefore by Lemma 2.4, we have

$$
\begin{aligned}
\sup _{z \in I_{\varepsilon}\left(e^{i t_{0}}\right) \backslash \operatorname{supp}(\psi)}\left|\psi^{* \prime}(z)\right| & =\sup _{z \in I_{\varepsilon}\left(e^{i t_{0}}\right) \backslash \operatorname{supp}(\psi)}\left(\left|\psi_{1}^{* \prime}(z)\right|+\left|\psi_{2}^{* \prime}(z)\right|\right) \\
& \geq \sup _{z \in I_{\varepsilon}\left(e^{i t_{0}}\right) \backslash \operatorname{supp}(\psi)}\left|\psi_{1}^{* \prime}(z)\right|=\infty .
\end{aligned}
$$

We have $\varphi=\tau_{-\lambda} \circ \psi$ and $\varphi^{* \prime}=\psi^{* \prime}\left(\tau_{-\lambda}^{\prime} \circ \psi^{*}\right)$ on $I_{\varepsilon}\left(e^{i t_{0}}\right) \backslash \operatorname{supp}(\psi)$. Since $\inf _{z \in \partial \mathbb{D}}\left|\tau_{-\lambda}^{\prime}(z)\right|>0$, we have $\sup _{z \in I_{\varepsilon}\left(e^{i t_{0}}\right) \backslash \operatorname{supp}(\psi)}\left|\varphi^{* \prime}(z)\right|=\infty$. Since $\operatorname{supp}(\varphi)=$ $\operatorname{supp}(\psi)$, we get the assertion. 
By Lemma 2.2 and Theorem 2.5, we have the following theorem.

Theorem 2.6. Let $\varphi$ be an inner function. If $\operatorname{supp}(\varphi) \neq \overline{i n t \operatorname{supp}(\varphi)}$, then there exists a function $u \in H^{2} \backslash H^{\infty}$ such that $M_{u} C_{\varphi}: H^{2} \rightarrow H^{2}$ is bounded.

There are many examples of inner functions $\varphi$ such that $\operatorname{supp}(\varphi)=\overline{\operatorname{int} \operatorname{supp}(\varphi)}$ and there exists a function $u \in H^{2} \backslash H^{\infty}$ such that $M_{u} C_{\varphi}: H^{2} \rightarrow H^{2}$ is bounded. For example, let $\varphi_{1}$ be an inner function satisfying $\operatorname{supp}\left(\varphi_{1}\right)=\left\{e^{i t}: 0 \leq t \leq \pi\right\}$. Let

$$
\varphi_{2}(z)=\exp \left(\frac{z+1}{z-1}+\frac{z-1}{z+1}\right) .
$$

Then $\varphi_{2}$ is a singular inner function satisfying $\operatorname{supp}\left(\varphi_{2}\right)=\{1,-1\}$. Put $\varphi=\varphi_{1} \varphi_{2}$. Then we have that $\operatorname{supp}(\varphi)=\left\{e^{i t}: 0 \leq t \leq \pi\right\}$ and

$$
\sup _{\pi<t<2 \pi}\left|\varphi^{* \prime}\left(e^{i t}\right)\right|=\infty .
$$

Hence there exists a function $u \in H^{2} \backslash H^{\infty}$ such that $M_{u} C_{\varphi}: H^{2} \rightarrow H^{2}$ is bounded.

Let $\psi$ be an inner function with $\operatorname{supp}(\psi)=\partial \mathbb{D}$. By the above fact, $C_{\psi}\left(M_{u} C_{\varphi}\right)=$ $M_{u \circ \psi} C_{\varphi \circ \psi}$ is bounded on $H^{2}$. We have that $\operatorname{supp}(\varphi \circ \psi)=\partial \mathbb{D}$ and $u \circ \psi \in H^{2} \backslash H^{\infty}$. Hence there are an inner function $\eta$ with $\operatorname{supp}(\eta)=\partial \mathbb{D}$ and $v \in H^{2} \backslash H^{\infty}$ such that $M_{v} C_{\eta}: H^{2} \rightarrow H^{2}$ is bounded.

We shall give another sufficient condition. One may check the following easily.

Lemma 2.7. Let $\varphi_{1}, \varphi_{2}$ be inner functions and $I$ be an open subarc of $\partial \mathbb{D}$ such that $I \cap \operatorname{supp}\left(\varphi_{1} \varphi_{2}\right)=\emptyset$. Then $m\left(\varphi_{1}^{*}(I)\right) \leq m\left(\left(\varphi_{1} \varphi_{2}\right)^{*}(I)\right)$.

Lemma 2.8. Let $\varphi$ be an inner function and $I$ be an open subarc of $\partial \mathbb{D}$ such that $I \cap \operatorname{supp}(\varphi)=\emptyset$. We write $I=\left\{e^{i t}: t_{1}<t<t_{2}\right\}$. Let $\sigma(t)$ be an extended real valued continuous function on $\left[t_{1}, t_{2}\right]$ such that $\varphi^{*}\left(e^{i t}\right)=e^{i \sigma(t)}, \sigma(t)$ is differentiable and $\sigma^{\prime}(t)>0$ on $\left(t_{1}, t_{2}\right)$. If $\sigma\left(t_{2}\right)-\sigma\left(t_{1}\right)<\infty$, then for each $\varepsilon>0$, there is an inner function $\psi$ such that $\varphi / \psi$ is inner, $\operatorname{supp}(\psi)=\operatorname{supp}(\varphi)$ and $m\left(\psi^{*}(I)\right)<\varepsilon$.

Proof. By the assumption, $-\infty<\sigma\left(t_{1}\right)<\sigma\left(t_{2}\right)<\infty$. Take a positive integer $n$ satisfying $\left(\sigma\left(t_{2}\right)-\sigma\left(t_{1}\right)\right) / 2 n \pi<\varepsilon$. It is not difficult to see the existence of inner functions $\varphi_{1}, \varphi_{2}, \cdots, \varphi_{n}$ such that $\varphi=\varphi_{1} \varphi_{2} \cdots \varphi_{n}$ and $\operatorname{supp}\left(\varphi_{j}\right)=\operatorname{supp}(\varphi)$ for every $1 \leq j \leq n$. For each $1 \leq j \leq n$, there is a real valued continuous function $\sigma_{j}(t)$ on $\left[t_{1}, t_{2}\right]$ such that $\varphi_{j}^{*}\left(e^{i t}\right)=e^{i \sigma_{j}(t)}, \sigma_{j}(t)$ is differentiable and $\sigma_{j}^{\prime}(t)>0$ on $\left(t_{1}, t_{2}\right)$. We have

$$
\sigma\left(t_{2}\right)-\sigma\left(t_{1}\right)=\sum_{j=1}^{n}\left(\sigma_{j}\left(t_{2}\right)-\sigma_{j}\left(t_{1}\right)\right) .
$$

Then $\left(\sigma_{j_{0}}\left(t_{2}\right)-\sigma_{j_{0}}\left(t_{1}\right)\right) / 2 \pi<\varepsilon$ for some $1 \leq j_{0} \leq n$. Hence $m\left(\varphi_{j_{0}}^{*}(I)\right)<\varepsilon$. Put $\psi=\varphi_{j_{0}}$. Then $\varphi / \psi$ is inner, $\operatorname{supp}(\psi)=\operatorname{supp}(\varphi)$ and $m\left(\psi^{*}(I)\right)<\varepsilon$.

For an inner function $\varphi$ and a measurable subset $E \subset \partial \mathbb{D}$, we put

$$
\varphi^{*(-1)}(E)=\left\{e^{i \theta} \in \partial \mathbb{D}: \varphi^{*}\left(e^{i \theta}\right) \in E\right\} .
$$


If $\varphi(0)=0$, then it is known that

$$
m\left(\varphi^{*(-1)}(E)\right)=m(E)
$$

for any measurable subset $E$ of $\partial \mathbb{D}$.

Theorem 2.9. Let $\varphi$ be an inner function. Suppose that there is a sequence of mutually disjoint open subarcs $\left\{I_{n}\right\}_{n \geq 1}$ of $\partial \mathbb{D}$ such that $\bigcup_{n=1}^{\infty} I_{n}=\partial \mathbb{D} \backslash \operatorname{supp}(\varphi)$. For each $n \geq 1$, let $I_{n}=\left\{e^{i t}: t_{n, 1}<t<t_{n, 2}\right\}$ and $\sigma_{n}(t)$ be an extended real valued continuous function on $\left[t_{n, 1}, t_{n, 2}\right]$ such that $\varphi_{n}^{*}\left(e^{i t}\right)=e^{i \sigma_{n}(t)}, \sigma_{n}(t)$ is differentiable and $\sigma_{n}^{\prime}(t)>0$ on $\left(t_{n, 1}, t_{n, 2}\right)$. Then we have the following.

(i) If $\sigma_{n}\left(t_{n, 2}\right)-\sigma_{n}\left(t_{n, 1}\right)=\infty$, then $\sup _{z \in I_{n}}\left|\varphi^{* \prime}(z)\right|=\infty$.

(ii) Suppose that $\sigma_{n}\left(t_{n, 2}\right)-\sigma_{n}\left(t_{n, 1}\right)<\infty$ for every $n \geq 1$. If $\sum_{n=1}^{\infty}\left(\sigma_{n}\left(t_{n, 2}\right)-\right.$ $\left.\sigma_{n}\left(t_{n, 1}\right)\right)=\infty$, then

$$
\sup _{z \in \partial \mathbb{D} \backslash \operatorname{supp}(\varphi)}\left|\varphi^{* \prime}(z)\right|=\infty .
$$

(iii) Suppose that $\sigma_{n}\left(t_{n, 2}\right)-\sigma_{n}\left(t_{n, 1}\right)<\infty$ for every $n \geq 1$. If $m(\operatorname{supp}(\varphi))=0$, then $\sum_{n=1}^{\infty}\left(\sigma_{n}\left(t_{n, 2}\right)-\sigma_{n}\left(t_{n, 1}\right)\right)=\infty$.

If one of the assumptions of (i), (ii) and (iii) holds, then there exists $u \in H^{2} \backslash H^{\infty}$ such that $M_{u} C_{\varphi}: H^{2} \rightarrow H^{2}$ is bounded.

Proof. (i) follows from the mean valued theorem.

(ii) For each positive integer $j$, there is $n_{j}$ such that

$$
j \leq \frac{\sigma_{n_{j}}\left(t_{n_{j}, 2}\right)-\sigma_{n_{j}}\left(t_{n_{j}, 1}\right)}{t_{n_{j}, 2}-t_{n_{j}, 1}} .
$$

For, if not, then there is $j_{0}$ such that

$$
\frac{\sigma_{n}\left(t_{n, 2}\right)-\sigma_{n}\left(t_{n, 1}\right)}{t_{n, 2}-t_{n, 1}}<j_{0}
$$

for every $n \geq 1$. Then we have

$$
\infty=\sum_{n=1}^{\infty}\left(\sigma_{n}\left(t_{n, 2}\right)-\sigma_{n}\left(t_{n, 1}\right)\right)<j_{0} \sum_{n=1}^{\infty}\left(t_{n, 2}-t_{n, 1}\right) \leq 2 \pi j_{0} .
$$

This is a contradiction.

By the mean valued theorem, there is $t_{n_{j}, 1}<\theta_{j}<t_{n_{j}, 2}$ satisfying $j \leq \sigma_{n_{j}}^{\prime}\left(\theta_{j}\right)=$ $\left|\varphi^{* \prime}\left(e^{i \theta_{j}}\right)\right|$ for every $j$. Therefore we get

$$
\sup _{z \in \partial \mathbb{D} \backslash \operatorname{supp}(\varphi)}\left|\varphi^{* \prime}(z)\right|=\sup _{n \geq 1} \sup _{z \in I_{n}}\left|\varphi^{* \prime}(z)\right|=\infty .
$$

(iii) To prove (iii), suppose that $\sum_{n=1}^{\infty}\left(\sigma_{n}\left(t_{n, 2}\right)-\sigma_{n}\left(t_{n, 1}\right)\right)<\infty$. We shall lead a contradiction. By the assumption, there is $n_{0}$ such that $\sum_{n=n_{0}}^{\infty}\left(\sigma_{n}\left(t_{n, 2}\right)-\right.$ $\left.\sigma_{n}\left(t_{n, 1}\right)\right)<1$. By Lemmas 2.7 and 2.8, there is an inner function $\psi$ such that $\varphi / \psi$ is inner, $\operatorname{supp}(\psi)=\operatorname{supp}(\varphi)$ and $\sum_{n=1}^{\infty} m\left(\psi^{*}\left(I_{n}\right)\right)<1$. Therefore

$$
m\left(\psi^{*}\left(\bigcup_{n=1}^{\infty} I_{n}\right)\right)=m\left(\bigcup_{n=1}^{\infty} \psi^{*}\left(I_{n}\right)\right) \leq \sum_{n=1}^{\infty} m\left(\psi^{*}\left(I_{n}\right)\right)<1 .
$$


Let $\lambda=\psi(0)$ and $\tau_{\lambda}(z)=(z-\lambda) /(1-\bar{\lambda} z)$. Set $\eta(z)=\tau_{\lambda} \circ \psi$. Since $\tau_{\lambda}$ is an automorphism, $m(E)=0$ if and only if $m\left(\tau_{\lambda}^{*}(E)\right)=0$ for every measurable subset $E$ of $\partial \mathbb{D}$. Then we have

$$
m\left(\eta^{*}\left(\bigcup_{n=1}^{\infty} I_{n}\right)\right)=m\left(\tau_{\lambda}^{*}\left(\psi^{*}\left(\bigcup_{n=1}^{\infty} I_{n}\right)\right)\right)<1 .
$$

Since $\eta$ is an inner function and $\eta(0)=0$, we have

$$
m\left(\bigcup_{n=1}^{\infty} I_{n}\right) \leq m\left(\eta^{*(-1)}\left(\eta^{*}\left(\bigcup_{n=1}^{\infty} I_{n}\right)\right)\right)=m\left(\eta^{*}\left(\bigcup_{n=1}^{\infty} I_{n}\right)\right)<1 .
$$

Since $m(\operatorname{supp}(\varphi))=0$, we have $m\left(\bigcup_{n=1}^{\infty} I_{n}\right)=1$. Thus we get a contradiction.

The last part of the assertion follows from Lemma 2.2.

\section{OTHER SUFFICIENT CONDITIONS}

For an inner function $\varphi$, first we consider the following condition.

$(\alpha)$ There is a sequence of mutually disjoint measurable subsets $\left\{C_{n}\right\}_{n \geq 1}$ of $\partial \mathbb{D}$ and a sequence of positive numbers $\left\{\delta_{n}\right\}_{n \geq 1}$ satisfying $\sum_{n=1}^{\infty} \delta_{n}<\infty$ such that $m\left(C_{n}\right)>0$ and $m\left(C_{n} \cap \varphi^{*(-1)}(E)\right) \leq \delta_{n} m(E)$ for every measurable subset $E$ of $\partial \mathbb{D}$ and for every $n \geq 1$.

We do not know whether condition $(\alpha)$ holds or not for any inner function $\varphi$ satisfying $m(\operatorname{supp}(\varphi))>0$. We shall show the following theorem.

Theorem 3.1. Let $\varphi$ be an inner function satisfying condition $(\alpha)$. Then there is $u \in H^{2} \backslash H^{\infty}$ such that $M_{u} C_{\varphi}: H^{2} \rightarrow H^{2}$ is bounded.

Proof. Since $\left\{C_{n}\right\}_{n \geq 1}$ is a sequence of mutually disjoint measurable subsets of $\partial \mathbb{D}$, we have $\sum_{n=1}^{\infty} m\left(C_{n}\right) \leq m(\partial \mathbb{D})=1$. Then there is a sequence of positive numbers $\left\{a_{n}\right\}_{n \geq 1}$ such that $a_{n} \geq 1$ for every $n$,

$$
\sum_{n=1}^{\infty} a_{n} m\left(C_{n}\right)<\infty
$$

and $a_{n} \rightarrow \infty$ as $n \rightarrow \infty$. Since $\sum_{n=1}^{\infty} \delta_{n}<\infty$, moreover we may assume that

$$
\sum_{n=1}^{\infty} a_{n} \delta_{n}<\infty
$$

Put $a_{0}=1$ and $C_{0}=\partial \mathbb{D} \backslash \bigcup_{n=1}^{\infty} C_{n}$. Let $\eta$ be the function on $\partial \mathbb{D}$ defined by $\eta=a_{n}$ on $C_{n}$ for every $n \geq 0$. Then $\eta \geq 1$ on $\partial \mathbb{D}$ and

$$
\int_{\partial \mathbb{D}} \eta d m=\sum_{n=0}^{\infty} a_{n} m\left(C_{n}\right)<\infty .
$$

By [4, p. 53], there exists $u \in H^{2}$ such that $|u|^{2}=\eta$ a.e. on $\partial \mathbb{D}$. Since $a_{n} \rightarrow \infty$ as $n \rightarrow \infty$ and $m\left(C_{n}\right)>0$ for every $n \geq 1$, we have $u \notin H^{\infty}$. 
Let $\mathcal{L}$ be the set of measurable simple functions on $\partial \mathbb{D}$. Let $f \in \mathcal{L}$. We may write

$$
f=\sum_{i=1}^{\ell} c_{i} \chi_{\Lambda_{i}},
$$

where $m\left(\Lambda_{i}\right)>0$ for every $i$ and $\Lambda_{i} \cap \Lambda_{j}=\emptyset$ for $i \neq j$. We have

$$
\begin{aligned}
\left\|M_{u} C_{\varphi} f\right\|_{2}^{2} & =\int_{\partial \mathbb{D}}|u|^{2}\left|f \circ \varphi^{*}\right|^{2} d m=\sum_{n=0}^{\infty} a_{n} \int_{C_{n}}\left|f \circ \varphi^{*}\right|^{2} d m \\
& =\int_{C_{0}}\left|f \circ \varphi^{*}\right|^{2} d m+\sum_{n=1}^{\infty} a_{n} \sum_{i=1}^{\ell}\left|c_{i}\right|^{2} \int_{C_{n}} \chi_{\Lambda_{i}} \circ \varphi^{*} d m \\
& \leq \int_{\partial \mathbb{D}}\left|f \circ \varphi^{*}\right|^{2} d m+\sum_{n=1}^{\infty} a_{n} \sum_{i=1}^{\ell}\left|c_{i}\right|^{2} m\left(C_{n} \cap \varphi^{*(-1)}\left(\Lambda_{i}\right)\right) \\
& \leq\left\|C_{\varphi}\right\|^{2}\|f\|_{2}^{2}+\sum_{n=1}^{\infty} a_{n} \delta_{n} \sum_{i=1}^{\ell}\left|c_{i}\right|^{2} m\left(\Lambda_{i}\right) \quad \text { by condition }(\alpha) \\
& =\left(\left\|C_{\varphi}\right\|^{2}+\sum_{n=1}^{\infty} a_{n} \delta_{n}\right)\|f\|_{2}^{2}
\end{aligned}
$$

Since $\sum_{n=1}^{\infty} a_{n} \delta_{n}<\infty, M_{u} C_{\varphi}: \mathcal{L} \rightarrow L^{2}(\partial \mathbb{D})$ is a bounded linear map. Since $\mathcal{L}$ is dense in $L^{2}(\partial \mathbb{D}), M_{u} C_{\varphi}$ may be extended boundedly on $L^{2}(\partial \mathbb{D})$. Thus $M_{u} C_{\varphi}: H^{2} \rightarrow H^{2}$ is bounded.

We shall give a sufficient condition on an inner function $\varphi$ for which satisfies condition $(\alpha)$. We consider the following condition for $\varphi$.

$(\beta)$ There is a sequence of mutually disjoint measurable subsets $\left\{E_{n}\right\}_{n \geq 1}$ of $\partial \mathbb{D}$ such that $m\left(E_{n} \cap \varphi^{*(-1)}(E)\right)>0$ for every measurable subset $E$ of $\partial \mathbb{D}$ satisfying $m(E)>0$ and for every $n \geq 1$.

We do not know whether condition $(\beta)$ holds or not for any inner function $\varphi$ satisfying $m(\operatorname{supp}(\varphi))>0$.

Theorem 3.2. If an inner function $\varphi$ satisfies condition $(\beta)$, then $\varphi$ satisfies condition $(\alpha)$.

Proof. We divide the proof into two cases.

Case 1. Suppose that $\varphi(0)=0$. Then it is known that

$$
m\left(\varphi^{*(-1)}(E)\right)=m(E)
$$

for any measurable subset $E$ of $\partial \mathbb{D}$. By condition $(\beta)$, there is a family of mutually disjoint measurable subsets $\left\{E_{n, j}: 1 \leq n, 1 \leq j \leq N_{n}\right\}$ of $\partial \mathbb{D}$ such that

$$
m\left(E_{n, j} \cap \varphi^{*(-1)}(E)\right)>0
$$


for every measurable subset $E$ of $\partial \mathbb{D}$ satisfying $m(E)>0$ and for every $n \geq$ $1,1 \leq j \leq N_{n}$. Moreover we may assume that

$$
\sum_{n=1}^{\infty} \frac{1}{N_{n}}<\infty
$$

For each $n \geq 1$, let $W_{n}=\bigcup_{j=1}^{N_{n}} E_{n, j}$. Then

$$
\left\{W_{n}\right\}_{n \geq 1} \text { is a sequence of mutually disjoint sets. }
$$

Put

$$
\mu_{n, j}(E)=m\left(E_{n, j} \cap \varphi^{*(-1)}(E)\right)
$$

for every measurable subset $E$ of $\partial \mathbb{D}$. Then $\mu_{n, j}$ is a positive measure on $\partial \mathbb{D}$. By (3.1), we have $\mu_{n, j} \ll m$, so there is a nonnegative integrable function $f_{n, j}$ on $\partial \mathbb{D}$ such that

$$
\int_{E} f_{n, j} d m=m\left(E_{n, j} \cap \varphi^{*(-1)}(E)\right) .
$$

By (3.1) again, we have

$$
\int_{E} \sum_{j=1}^{N_{n}} f_{n, j} d m=m\left(W_{n} \cap \varphi^{*(-1)}(E)\right) \leq m\left(\varphi^{*(-1)}(E)\right)=m(E)
$$

for every measurable subset $E$ of $\partial \mathbb{D}$. Hence

$$
0 \leq \sum_{j=1}^{N_{n}} f_{n, j} \leq 1 \quad \text { a.e. on } \partial \mathbb{D}
$$

Let

$$
A_{n, j}=\left\{e^{i \theta} \in \partial \mathbb{D}: f_{n, j}\left(e^{i \theta}\right) \leq \frac{1}{N_{n}}\right\} .
$$

By (3.6) and (3.7), we have

$$
m\left(\partial \mathbb{D} \backslash \bigcup_{j=1}^{N_{n}} A_{n, j}\right)=0
$$

Let

$$
B_{n, 1}=A_{n, 1}, \quad B_{n, j}=A_{n, j} \backslash \bigcup_{i=1}^{j-1} A_{n, i}\left(2 \leq j \leq N_{n}\right)
$$

Then

$$
\left\{B_{n, j}: 1 \leq j \leq N_{n}\right\} \text { is a set of mutually disjoint sets }
$$

and

$$
m\left(\bigcup_{j=1}^{N_{n}} B_{n, j}\right)=m\left(\bigcup_{j=1}^{N_{n}} A_{n, j}\right)=1
$$

We have that

$$
m\left(B_{n, j}\right)>0 \quad \text { for some } 1 \leq j \leq N_{n} .
$$


For a measurable subset $E$ of $B_{n, j}$, we have

$$
\begin{aligned}
m\left(E_{n, j} \cap \varphi^{*(-1)}(E)\right) & =\int_{E} f_{n, j} d m \quad \text { by }(3.5) \\
& \leq \frac{m(E)}{N_{n}} \quad \text { by }(3.7) \text { and }(3.8) .
\end{aligned}
$$

Hence

$$
m\left(E_{n, j} \cap \varphi^{*(-1)}(E)\right) \leq \frac{m(E)}{N_{n}} \quad \text { for every } E \subset B_{n, j}
$$

For each $1 \leq j \leq N_{n}$, let

$$
C_{n, j}=E_{n, j} \cap \varphi^{*(-1)}\left(B_{n, j}\right)
$$

and for each $n \geq 1$, set $C_{n}=\bigcup_{j=1}^{N_{n}} C_{n, j}$. Then $C_{n} \subset W_{n}$ and by (3.4), $\left\{C_{n}\right\}_{n \geq 1}$ is a sequence of mutually disjoint sets. For each $n \geq 1$, we have

$$
\begin{aligned}
m\left(C_{n}\right) & =\sum_{j=1}^{N_{n}} m\left(C_{n, j}\right) \\
& =\sum_{j=1}^{N_{n}} m\left(E_{n, j} \cap \varphi^{*(-1)}\left(B_{n, j}\right)\right) \quad \text { by }(3.13) \\
& >0 \quad \text { by }(3.2) \text { and }(3.11) .
\end{aligned}
$$

Hence $m\left(C_{n}\right)>0$ for every $n \geq 1$.

For a measurable subset $E$ of $\partial \mathbb{D}$ and $n \geq 1$, we have

$$
\begin{aligned}
m\left(C_{n} \cap \varphi^{*(-1)}(E)\right) & =\sum_{j=1}^{N_{n}} m\left(C_{n, j} \cap \varphi^{*(-1)}(E)\right) \\
& =\sum_{j=1}^{N_{n}} \sum_{k=1}^{N_{n}} m\left(C_{n, j} \cap \varphi^{*(-1)}\left(B_{n, k} \cap E\right)\right)
\end{aligned}
$$

by (3.9) and (3.10) 


$$
\begin{aligned}
& =\sum_{j=1}^{N_{n}} \sum_{k=1}^{N_{n}} m\left(E_{n, j} \cap \varphi^{*(-1)}\left(B_{n, j}\right)\right. \\
& \left.\cap \varphi^{*(-1)}\left(B_{n, k} \cap E\right)\right) \quad \text { by (3.13) } \\
& =\sum_{j=1}^{N_{n}} m\left(E_{n, j} \cap \varphi^{*(-1)}\left(B_{n, j} \cap E\right)\right) \quad \text { by }(3.9) \\
& \leq \sum_{j=1}^{N_{n}} \frac{m\left(B_{n, j} \cap E\right)}{N_{n}} \quad \text { by }(3.12) \\
& =\frac{m(E)}{N_{n}} \quad \text { by (3.9) and }(3.10) .
\end{aligned}
$$

Putting $\delta_{n}=1 / N_{n}>0$, we have $m\left(C_{n} \cap \varphi^{*(-1)}(E)\right) \leq \delta_{n} m(E)$. By (3.3), $\sum_{n=1}^{\infty} \delta_{n}<\infty$. Thus $\varphi$ satisfies condition $(\alpha)$.

Case 2. Suppose that $\lambda:=\varphi(0) \neq 0$. Let $\tau_{\lambda}(z)=(z-\lambda) /(1-\bar{\lambda} z)$ and $\psi=\tau_{\lambda} \circ \varphi$. We have $\psi^{*}=\tau_{\lambda}^{*} \circ \varphi^{*}$. For a measurable subset $E$ of $\partial \mathbb{D}$ with $m(E)>0, \psi^{*(-1)}(E)=\varphi^{*(-1)}\left(\tau_{\lambda}^{*(-1)}(E)\right)$. Since $m\left(\tau_{\lambda}^{*(-1)}(E)\right)>0$ and $\varphi$ satisfies condition $(\beta)$, we have

$$
m\left(E_{n} \cap \psi^{*(-1)}(E)\right)=m\left(E_{n} \cap \varphi^{*(-1)}\left(\tau_{\lambda}^{*(-1)}(E)\right)\right)>0
$$

for every $n \geq 1$. Hence $\psi$ satisfies condition $(\beta)$. By Case $1, \psi$ satisfies condition $(\alpha)$. Then there is a sequence of mutually disjoint measurable subsets $\left\{D_{n}\right\}_{n \geq 1}$ of $\partial \mathbb{D}$ and a sequence of positive numbers $\left\{\sigma_{n}\right\}_{n \geq 1}$ satisfying $\sum_{n=1}^{\infty} \sigma_{n}<\infty$ such that $m\left(D_{n}\right)>0$ and

$$
m\left(D_{n} \cap \psi^{*(-1)}(A)\right) \leq \sigma_{n} m(A)
$$

for every measurable subset $A$ of $\partial \mathbb{D}$ and for every $n \geq 1$. Since $\tau_{\lambda}$ is an automorphism, there is $K>0$ such that $m\left(\tau_{\lambda}^{*}(A)\right) \leq K m(A)$ for every $A$. We have $\psi^{*(-1)}\left(\tau_{\lambda}^{*}(A)\right)=\varphi^{*(-1)}(A)$ and

$$
\begin{aligned}
m\left(D_{n} \cap \varphi^{*(-1)}(A)\right) & =m\left(D_{n} \cap \psi^{*(-1)}\left(\tau_{\lambda}^{*}(A)\right)\right. \\
& \leq \sigma_{n} m\left(\tau_{\lambda}^{*}(A)\right) \leq \sigma_{n} K m(A) .
\end{aligned}
$$

Hence $\varphi$ satisfies condition $(\alpha)$.

By Theorems 3.1 and 3.2, we have the following.

Corollary 3.3. Let $\varphi$ be an inner function satisfying condition $(\beta)$. Then there is $u \in H^{2} \backslash H^{\infty}$ such that $M_{u} C_{\varphi}: H^{2} \rightarrow H^{2}$ is bounded.

Acknowledgement. The first author is partially supported by Grant-in-Aid for Scientific Research, Japan Society for the Promotion of Science (No.24540164).

\section{REFERENCES}

1. C.C. Cowen and B.D. MacCluer, Composition Operators on Spaces of Analytic Functions, Studies in Advanced Mathematics. CRC Press, Boca Raton, FL, 1995.

2. P. Duren, Theory of $H^{p}$ Spaces, Academic Press, New York, 1970. 
3. J.B. Garnett, Bounded Analytic Functions, Academic Press, New York, 1981.

4. K. Hoffman, Banach Spaces of Analytic Functions, Prentice-Hall, Englewood Cliffs, N.J., 1962.

5. K.J. Izuchi, Q.D. Nguyen and S. Ohno, Composition operators induced by analytic maps to the polydisk, Canadian J. Math. 64 (2012), 1329-1340.

6. J. Littlewood, On inequalities in the theory of function, Proc. London Math. Soc. (2) 23 (1925), 481-519.

7. A. Richards Jr, Isometries and composition-operators on Hardy spaces in connection with the measure-theoretic properties of inner-functions, Thesis, University of WisconsinMadison, 1974.

8. W. Rudin, Real and Complex Analysis, Third Edition, McGraw-Hill, New York, 1987.

9. J.H. Shapiro, Composition Operators and Classical Function Theory, Springer-Verlag, New York, 1993.

${ }^{1}$ Department of Mathematics, Nitgata University, Nimgata 950-2181, Japan.

E-mail address: izuchi@m.sc.niigata-u.ac.jp

2 Department of Mathematics, Faculty of Education, Yamaguchi University, YAMAGUCHI 753-8511, JAPAN.

E-mail address: izuchi@yamaguchi-u.ac.jp

3 Aoyama-Shinmachi 18-6-301, Niigata 950-2006, JaPAn.

E-mail address: yfd10198@nifty.com 